\title{
Letter from the Dean
}

It is at times difficult to appreciate the full spectrum of knowledge translation. The CIHR considers knowledge translation to reflect the iterative process that includes synthesis, dissemination, exchange and the ethically sound application of knowledge to improve the health of Canadians. Knowledge Translation Canada, a network of Canadian experts in knowledge translation, defines this as one of the greatest challenges in healthcare. They have synthesized this to reflect the gap in applying the results of health research to the patient bedside. This is often called the translational gap and has been an area of great discourse over the last many years. It is often suggested that the clinician scientist is critical to the closing of this gap and as such, it is the gradual decline in the number of clinician scientists being trained that is viewed as one of the greatest risks for knowledge translation. It is a gap that we as a medical school have taken a lead role in attempting to understand and then rectify, a role that has led to the generation of Canadian consensus recommendations for the training of clinician scientists. While these recommendations cover a broad range of training paradigms, the goal is clearly to improve the health of Canadians through not only the genesis of new knowledge but its application at the bedside. In this light, it truly is the responsibility of all clinicians to understand knowledge translation and to apply its principles in patient management.

Many would argue that the existing definitions of knowledge translation may be somewhat restrictive. Increasingly, as we understand the complex relationship between health outcomes and fundamental cellular or molecular mechanisms of disease, the ability to effectively communicate our understanding of the disease process and then apply this to the bedside has become equally as challenging and complex. As we must move increasingly towards cost containment across virtually all developed nations who expend a significant proportion of their gross national product on health care, it is critical that the next generation of medical leaders be understanding of the barriers to knowledge translation and how overcoming these can improve health outcomes. As individual clinicians and as members of health care teams, it will fall increasingly to us to ensure that this knowledge is used to ensure a sustainable healthcare system. Quite simply put, the health care system in which we exist currently is not sustainable in its current form. It will be for your generation of health care providers to have a critical understanding of not only disease processes but also to be able to critically appraise biomedical innovations and emerging healthcare practices. It will fall to you to then integrate this understanding in a manner that ensures excellent healthcare for Canadians with cost containment. It would be fair to say that innovations brought into the healthcare arena today rarely are less expensive than their predecessors and indeed many of the more complex treatments for previously refractory diseases carry a tremendous financial cost. It will be up to this next generation of healthcare practitioners, including yourselves, to be able to critically appraise the literature of these advances and then help to influence not only their use at the bedside, but how that are integrated into a much broader Canadian healthcare milieu.

The UWO Medical Journal has a long and rich tradition of helping to foster the development of this skill set. Written and supported by students, often in the early stages of their career, this is an inspiring effort. I would encourage you to consider contributing to this journal by submitting articles which address this issue of knowledge translation. Not only is this a great benefit to your colleagues, but the exercise of undergoing peer review of manuscripts can be richly rewarding.

At a time when all aspects of science are under greater scrutiny, your ability to defend your decisions and particularly those that lead to alterations in the therapy of your patients, will be critical. The UWO Medical Journal is a key piece of this.

Michael J Strong, MD, FRCPC, FAAN, FCAHS Dean, Schulich School of Medicine \& Dentistry Distinguished University Professor 